\title{
Spectroscopy of near-Earth asteroids ${ }^{\star}$
}

\author{
R. Michelsen ${ }^{1}$, A. Nathues ${ }^{2}$, and C.-I. Lagerkvist ${ }^{3}$ \\ 1 Niels Bohr Institute, University of Copenhagen, Juliane Maries Vej 30, 2100 Copenhagen, Denmark \\ e-mail: rene@astro.ku.dk \\ 2 Max-Planck-Institut für Sonnensystemforschung, Max-Planck-Strasse 2, 37191 Katlenburg-Lindau, Germany \\ e-mail: nathues@linmpi.mpg.de \\ 3 Astronomical Observatory, Box 515, 75120 Uppsala, Sweden \\ e-mail: Claes-Ingvar.Lagerkvist@astro.uu.se
}

Received 15 March 2004 / Accepted 2 November 2005

\section{ABSTRACT}

We present spectra and taxonomic classifications of 12 Near-Earth Asteroids (NEAs) and 2 inner Main Belt asteroids. The observations were carried out with the ESO $3.5 \mathrm{~m}$ NTT and the Danish $1.54 \mathrm{~m}$ telescope at La Silla, Chile. Eleven of the investigated NEAs belong to the S class while only one C-type has been identified. Two NEAs were observed at phase angles larger than $60^{\circ}$ introducing significant phase reddening. In order to allow for comparisons between spectra of asteroids observed at different phase angles we make attempts to correct for this effect. However, it turned out that a uniform approach based on previous measurements of phase reddening by means of observational data is not reasonable. For the NEAs we question the approach of taxonomic classification in terms of subgroups, when the classification is based on spectra obtained at large phase angles.

Key words. minor planets, asteroids

\section{Introduction}

Near-Earth Asteroids (NEAs) have in the recent years obtained increasing attention due to the potential hazard these asteroids constitute for life on Earth (Morrison et al. 2002). Collisional phenomena played and play until today an important role for the development of the Solar System (Davis et al. 2002). Furthermore, NEAs are in several aspects of fundamental importance. The many meteorites found on Earth originate from at least 135 mineralogically different compositions (Meibom \& Clark 1999). This diversity among the meteorites has its origin in the fragmentation of primitive and differentiated asteroids in the Main Belt, parts of which by time are transported to the inner parts of the Solar System by various mechanisms (Nesvorný et al. 2002; Bottke et al. 2002). Fragments of these meteoritic parent bodies can hence be found among the population of NEAs, with an expected reflection of the meteoritic diversity among these asteroids. The link between meteorites and asteroids is however poorly determined, only in the case of the HED meteorites and the V-type asteroids (Binzel \& Xu 1993) a connection has been firmly assessed. The most likely explanation for these missing links is that the relatively large

^ Based on observations collected with the NTT telescope at the European Southern Observatory, Chile, proposal 70.C-0060. Based on observations collected with the Danish 1.54 m telescope, through grants from the Danish Natural Science Research Council.
Main Belt asteroids we try to link with meteorites have been subject to surface alterations that change the spectral characteristics of the surface material. Space weathering, i.e. solar irradiation and micro meteoritic bombardment, as well as grain size differences (Clark et al. 2002a), lead to spectral profile deviations from a fresh cut meteoritic surface. The meteoritic parent bodies may thus be more readily recognized among the smaller asteroid fragments, presumably with relatively young and less altered, almost dust free, surfaces (Haack et al. 2002). Due to the presence of NEAs in the vicinity of the Earth, even the smallest known members of this population provide an opportunity for spectroscopic observations with the aim of taxonomic classification. Thus NEAs are expected to be of vital importance for the identification of the missing links between Main Belt asteroids and meteorites. Reviews of the observational evidence for this scenario can be found in Lupishko \& Di Martino (1998) and Binzel et al. (2002). Some of the first to perform CCD reflectance spectroscopy for NEA investigations were Luu \& Jewitt (1990). Most of the NEAs observed by them appear to be of S-type, indicating an origin in the inner region of the asteroid belt. These asteroids are presumably the parent bodies of the ordinary chondrite meteorites (Binzel et al. (1996). In general NEAs appear to be highly taxonomic diverse as is evident from Binzel et al. (2001) and Angeli \& Lazarro (2002). This diversity is probably linked to the diversity among the meteorites, however, as only approx. $10 \%$ of the known 
Table 1. Objects and observational circumstances. The phase angle is denoted by $\alpha$, mag. is the $V$-magnitude at the time of observation, and $H$ is the absolute magnitude of the asteroids.

\begin{tabular}{|c|c|c|c|c|c|c|c|c|}
\hline Object & Orbit & Date & $\begin{array}{l}\text { Telescope and } \\
\text { instrument }\end{array}$ & $\begin{array}{l}\alpha \\
\left({ }^{\circ}\right)\end{array}$ & $\begin{array}{l}\text { mag. } \\
\text { (V) }\end{array}$ & $H$ & Solar analog & Method \\
\hline \multirow[t]{2}{*}{ (2329) Orthos } & $\mathrm{AP}$ & 02 Jan. 2003 & NTT/EMMI & 39.8 & 17.8 & 14.9 & HD 44594 & $900 \mathrm{~s}$ \\
\hline & & & & & & & HD 44594 & $900 \mathrm{~s}$ \\
\hline \multirow[t]{2}{*}{$2002 \mathrm{AG}_{29}$} & $\mathrm{AP}$ & 02 Jan. 2003 & NTT/EMMI & 38.4 & 19.0 & 18.6 & HD 44594 & B \\
\hline & & & & & & & HD 44594 & $A B$ \\
\hline (4957) Brucemurray & $\mathrm{AM}$ & 02 Jan. 2003 & NTT/EMMI & 27.8 & 17.4 & 15.1 & HD 44594 & A \\
\hline (35107) $1991 \mathrm{VH}$ & $\mathrm{AP}$ & 02 Jan. 2003 & NTT/EMMI & 39.8 & 16.6 & 16.9 & HD 44594 & $A B$ \\
\hline $2002 \mathrm{NY}_{31}$ & $\mathrm{AM}$ & 02 Jan. 2003 & NTT/EMMI & 45.7 & 17.1 & 17.3 & HD 44594 & ABBA \\
\hline (1917) Cuyo & $\mathrm{AM}$ & 02 Jan. 2003 & NTT/EMMI & 24.8 & 18.1 & 13.9 & HD4 4594 & $A B$ \\
\hline (25916) $2001 \mathrm{CP}_{44}$ & $\mathrm{AM}$ & 03 Jan. 2003 & NTT/EMMI & 17.0 & 18.1 & 13.3 & L102-1081 & $A B$ \\
\hline $2002 \mathrm{QE}_{15}$ & $\mathrm{AM}$ & 03 Jan. 2003 & NTT/EMMI & 49.1 & 17.4 & 16.3 & L102-1081 & BA \\
\hline $2002 \mathrm{DB}_{4}$ & AT & 03 Jan. 2003 & NTT/EMMI & 62.3 & 17.1 & 16.4 & L102-1081 & $A B$ \\
\hline $2002 \mathrm{~TB}_{58}$ & $\mathrm{AM}$ & 03 Jan. 2003 & NTT/EMMI & 60.2 & 17.7 & 16.5 & L102-1081 & B \\
\hline (914) Palisana & $q=1.93$ & 13 Feb. 2003 & DK1p54/DFOSC & 18.4 & 13.4 & 8.76 & HD 44594 & $3600 \mathrm{~s}$ \\
\hline \multirow[t]{2}{*}{ (393) Lampetia } & $q=1.86$ & 13 Feb. 2003 & DK1p54/DFOSC & 5.7 & 13.8 & 8.39 & HD 44594 & $2400 \mathrm{~s}$ \\
\hline & & & & & & & HD 44594 & $3600 \mathrm{~s}$ \\
\hline $1978 \mathrm{CA}$ & $\mathrm{AP}$ & 14 Feb. 2003 & DK1p54/DFOSC & 30.1 & 15.8 & 17.1 & HD 44594 & ABABAB \\
\hline (4954) Eric & $\mathrm{AM}$ & 15 Feb. 2003 & DK1p54/DFOSC & 21.4 & 17.4 & 12.6 & HD 44594 & $A B A B$ \\
\hline
\end{tabular}

NEAs have been taxonomic classified, the meteorite-NEA link is still not firmly based. One of the reasons for the lack of taxonomic classifications based on spectroscopy is that NEAs tend to be challenging to observe. The proximity of the NEAs imply that their apparent motions on the sky are larger than for Main Belt asteroids, thus drawing heavy demands on the telescope capabilities to accurately follow the asteroids located in the slit, sometimes exceeding what can be achieved by most telescopes. Here we present the observations of 12 NEAs and 2 inner Main Belt asteroids, of which the majority were made by applying a method that can circumvent the tracking problem of the telescopes to some degree. In Sect. 2 we describe the observations and the applied methods, in Sect. 3 we discuss the general results, and present a short discussion for each asteroid. In Sect. 4 we give summarize and draw some conclusions on phase reddening of NEAs.

\section{The observations}

The observations have been carried out at the ESO $3.5 \mathrm{~m}$ NTT and $1.54 \mathrm{~m}$ Danish Telescope, both located at La Silla, Chile. Table 1 summarizes the observational circumstances for each asteroids. The EMMI spectrometer was used in two nights in January 2003. The instrument was used in its red mode (RILD) allowing low-dispersion spectroscopy. Grism \#7, with 150 grooves per mm, was selected together with an order sorting filer (OG-530, ESO filter \#645) and a $5.0^{\prime \prime}$ wide long slit. The detector consisted of two CCDs (Zeus and Michelle, installed May 2002), arranged in a mosaic with $4096 \times 4096$ pixels, read by 4 amplifiers. The spatial resolution is $0.165^{\prime \prime}$ per pixel, the default is to read the mosaic in 2-by-2 bin mode providing a spectral resolution of $5.8 \AA$ per pixel $(R=280)$. With the combined sensitivity of the CCD, grism and filter we were able to cover the wavelength range from $5500 \AA$ to $9300 \AA$. The wavelength calibration was made with a Helium-Argon lamp.

The observations at the Danish 1.54 m telescope were undertaken during Danish national time. An initial run demonstrated problems with the differential guiding of the telescope. During a further run, we tried to exploit an alternative observing strategy, but unfortunately this run was interrupted by bad weather. During a third run we were finally able to achieve some spectra which are presented below. The Danish telescope was equipped with DFOSC, a combined spectrograph and camera, and was used with a $5.0^{\prime \prime}$ wide long-slit and a medium dispersive grism (\#5), with 300 grooves per mm. The mounted detector was the so called "Ringo" CCD, with $2048 \times 4096$ pixels, of which the DFOSC optics can only utilize approx. half the area. The spatial resolution is $0.39^{\prime \prime}$ per pixel, with a spectral resolution of $3.3 \AA$ per pixel $(R=870)$. The covered wavelength range is $\approx 5200-9000 \AA$, however, with heavy fringing longward of $7000 \AA$. The wavelength calibration was made by using a helium-neon lamp.

The NTT is capable of differential tracking, by specifying the desired proper motion of an object, which is translated into an additional tracking rate. The Danish telescope is able to perform differential guiding, where the proper motion is translated to a motion of the guide star on the guide probe screen. In the ideal case, the NTT system allows indefinite tracking of the object, whereas the duration of the differential guiding of the Danish telescope is restricted to the field of view of the guide probe camera. However, both telescopes showed 
insufficient tracking for the relatively fast-moving NEAs, particularly for those objects moving faster than $\approx 1^{\prime \prime} / \mathrm{min}$. Despite of using a wide slit (5.0"), none of the systems were able to retain an NEA aligned in the slit for more than about $10 \mathrm{~min}$. For the NTT, the nature of the problems was not identifiable by us. For the $1.54 \mathrm{~m}$ Danish telescope, experiments (including the two inner Main Belt asteroids, see below) showed that due to the non-linearity of the conversion from cross-motion rate to the motion of the guide probe, only the central position of the guide probe screen offers a stable differential guiding, in contrast to the recommendation that the entire central region of the screen can be used (Storm 1996). This means that the exposure time is highly constrained by the proper motion of the asteroid.

As these difficulties prevented long exposures of fastmoving asteroids, we devised an alternative observing strategy. The method was already applied for CCD spectroscopy by Nathues (2000) in order to optimize the sky background removal especially in wavelength ranges heavily affected by atmospheric conditions. Instead of making one long exposure (for the Danish telescope exposure times up to 60 min were required) with the asteroid placed at one slit position, the exposure was split into two exposures of each $10 \mathrm{~min}$ at two different slit positions $A$ and $B$. The two positions are carefully selected to be sufficient apart to allow a clear separation between both spectra. Under good atmospheric conditions the background will remain stable during the exposure sequence. Hence by subtracting $A$ from $B$ and $B$ from $A$, a very accurate background removal is achieved. To remove any residual background in each of the two A-B or B-A spectra a standard method for sky background removal was applied, where a first order polynomial is fitted to the remaining background below and above the spectrum, and then subtracted. In a further step, the spectra of a sequence are averaged to improve the signalto-noise ratio. Furthermore, it is possible to pair wise combine a sequence $A B A B A B$ of spectra, thus a final spectrum comparable to a single long exposure can be obtained. Of course, the read-out noise is, due to the multiple reads in the ABA... sequence, higher than for an equivalent single exposure, but this disadvantage is more than compensated by the high accuracy of the sky background and bias removal, especially for wavelengths longward of $\approx 8000 \AA$. This non-autonomous use of the nodding method has the further advantage of allowing comparatively short exposure times and therefore being less insensitive to the telescope differential tracking/guiding capabilities. We chose $10 \mathrm{~min}$ per exposure to comply with the stability of the differential tracking/guiding of the telescopes.

For all observations, it was attempted to align the slit along the parallactic angle, to avoid distortion of the spectrum, should the asteroid move partly out of the slit. For each night, bias frames, spectral sky (NTT) and spectral dome (Danish telescope) flat fields and lamp spectra were obtained. During the observing runs, high emphasis was made at obtaining spectra of the Solar Analog stars at similar air masses as the asteroids. However, particularly for the NTT the brightness of HD $44594(V=6.61)$ caused saturation problems at high elevations. Attempts to use short exposures of $1 \mathrm{~s}$ with a strongly defocused telescope were in vain, and hence an alternative star had to be used for these cases.



Fig. 1. Comparison of the observed solar analogs. The top normalized spectrum is a division of a spectrum of HD 44594 (air mass 1.35) with a spectrum of L102-1081 (air mass 1.15). The lower normalized spectrum is a division of FS 13 (air mass 1.16) with L102-1081.

Thus, for the solar calibration of the reflectance spectra we used the Solar Analogs HD 44594 (Hardorp 1978), and L102-1081 (Landolt 1973), which show nearly alike spectra (Fig. 1). A spectral comparison with FS 13 (A UKIRT standard), used as a Solar Analog for infrared wavelengths (Binzel et al. (2001), revealed strong deviations shortward of $9200 \AA$ from the two other stars.

The observed asteroids and the observational circumstances are listed in Table 1. For each asteroid we specify the orbit type (AP: Apollo, AM: Amor, AT: Aten, $q$ : perihelion distance), the date and telescope used, the phase angle and the apparent $V$-magnitude, the absolute magnitude $(H)$ and the Solar Analog used for calibration. The last column specifies the observational method. If a single long exposure was performed, the exposure time in seconds is given. If the $A B$ nodding was applied, the sequence of which the spectrum is composed is specified. In some cases, part of a nodding sequence was lost due to technical problems, as reflected in the stated sequences. For each A or B spectrum, an exposure time of $600 \mathrm{~s}$ was used in the general case. For example ABAB means that the spectrum consists of 4 pair wise added spectra, corresponding to a total exposure time of $2400 \mathrm{~s}$. The spectra are shown in Figs. 2 and 3 .

The following reduction steps have been performed with ESO-MIDAS to acquire a calibrated asteroid spectrum:

1. Subtraction of the raw spectra, A-B and B-A.

2. Division by a normalized flat field.

3. Median filtering for removal of cosmic hits.

4. Fitting and subtraction of the residual sky background.

5. Extraction of a 1-dimensional spectrum.

6. Wavelength calibration with the lamp spectrum.

7. Extinction correction using the La Silla mean extinction curve (Tüg 1977).

8. Averaging of $A$ and $B$ spectra.

9. Division by a Solar Analog spectrum that has been obtained at approximately the same air mass as the asteroid spectra.

10. Normalization to unity flux at $5500 \AA$. 

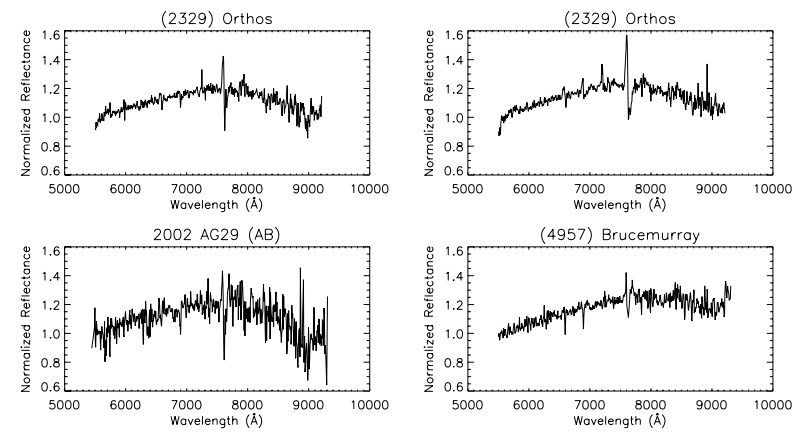

2002 NY3
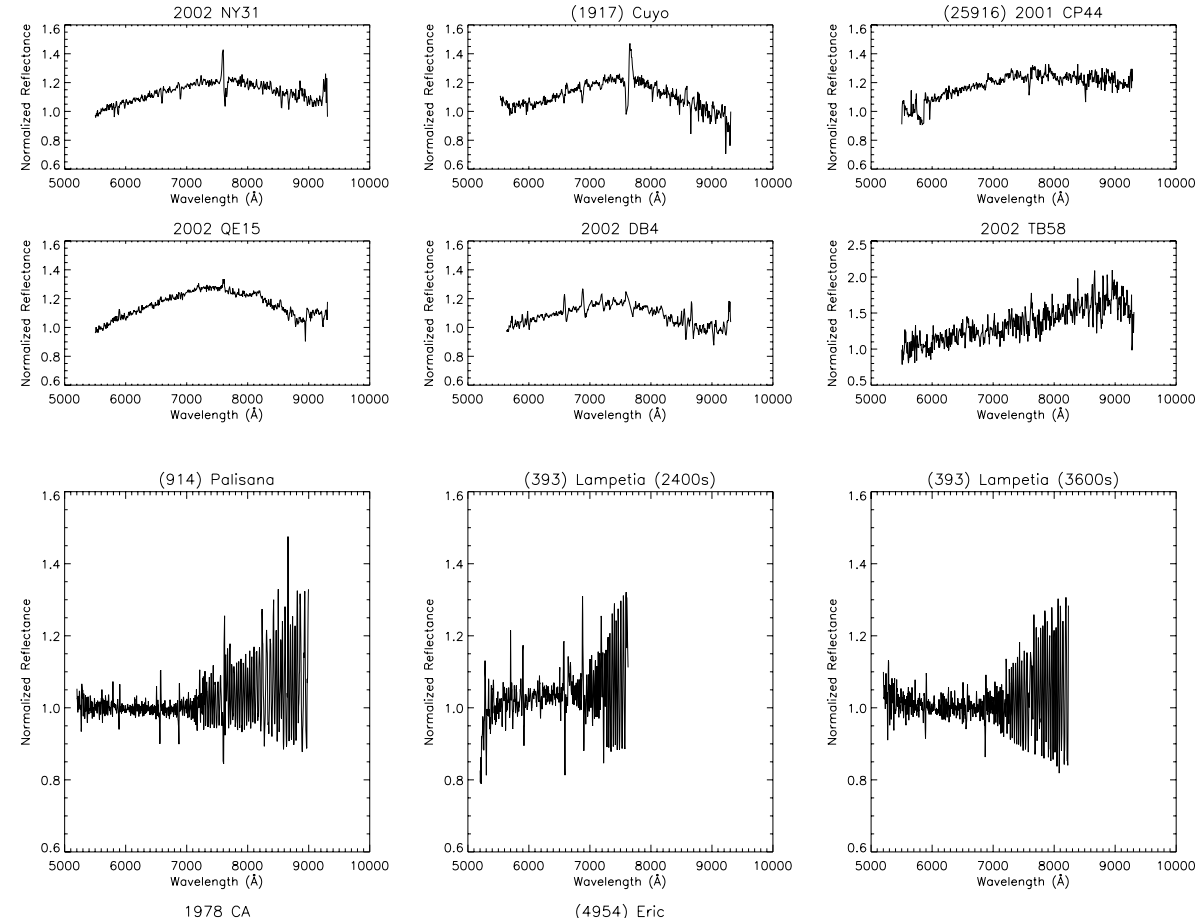
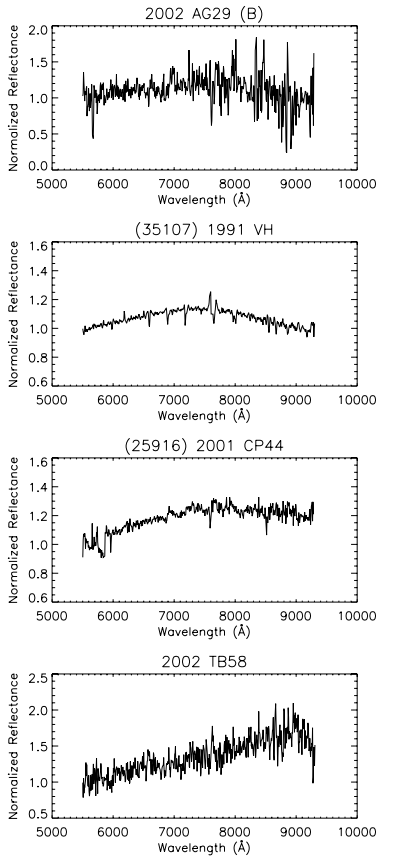

Fig. 2. Spectra obtained with the NTT (ESO) telescope. For 2002 AG29 two spectra were obtained from one single $B$ exposure and one $A B$ sequence. The spectra have been normalized to unity flux at $5500 \AA$.

Fig. 3. Spectra obtained with the Danish $1.54 \mathrm{~m}$ telescope. For (393) Lampetia two spectra were obtained from a $2400 \mathrm{~s}$ and a $3600 \mathrm{~s}$ exposure respectively, Table 1 . The spectra have been normalized to unity flux at $5500 \AA$.

Notice that for the $A B$ nodding it is not necessary to make a bias subtraction, as this is implicitly done in the first step. However, for some asteroids we have obtained only a single exposure hence utilizing the bias frames, and applying the standard sky background removal technique.

\section{Results}

The NTT spectra were truncated at $9300 \AA$, due to the systematic presence of artificial features longward of this wavelength. As one can see in Fig. 3 the spectra obtained with the Danish telescope are suffering from strong fringing beyond $7200 \AA$, hence for the most extreme cases the spectra have been truncated.
In Table 2 we present the achieved taxonomic classifications of the observed asteroids. $S^{\prime}$ is the spectral slope of the normalized spectra obtained from a least-square fit in the range 5000-7500 $\AA$ (Luu \& Jewitt 1990). When available, previous classifications are stated. Our classification is based on Tholen's taxonomic classification scheme (Tholen 1984; Tholen \& Barucci 1989), whereby some classifications must be taken as non-unique due to the absence of albedo information or/and the lack of spectral information longward of $7200 \AA$ as for (914) Palisana and (393) Lampetia (see Fig. 3).

As NEAs can come closer to the Earth than Main Belt asteroids, they are often observed at larger phase angles. But asteroids become redder with increasing phase angle, i.e. the spectral slope increases. This well-known effect is called "phase 
Table 2. Taxonomy of the observed asteroids. All the asteroids are NEAs except those marked with a $\uparrow$, these are Main Belt asteroids. The taxonomy marked with a $*$ is a best guess estimate, as the classification has been based on only a part of the visible spectrum (cf. Fig. 3). The references indicated in parentheses refer to 1: Tholen (1989); 2: Angeli \& Lazzaro (2002); 3: Lazzarin et al. (1997); 4: Binzel et al. (2004). See the text for further discussion.

\begin{tabular}{|c|c|c|c|}
\hline \multirow[t]{2}{*}{ Object } & \multirow{2}{*}{$\begin{array}{c}S^{\prime} \\
{[\% / 1000 \AA]}\end{array}$} & \multicolumn{2}{|c|}{ Taxonomy } \\
\hline & & Reference & This paper \\
\hline \multirow[t]{2}{*}{2329 Orthos } & $10.97 \pm 0.03$ & & $S$ \\
\hline & $13.08 \pm 0.03$ & & \\
\hline \multirow{2}{*}{$2002 \mathrm{AG}_{29}$} & $11.29 \pm 0.11$ & & $S$ \\
\hline & $11.60 \pm 0.06$ & & \\
\hline (4957) Brucemurray & $12.38 \pm 0.03$ & $\mathrm{Sl}^{(2)}$ & $S$ \\
\hline (35107) $1991 \mathrm{VH}$ & $7.70 \pm 0.02$ & $\mathrm{Sk}^{(4)}$ & S \\
\hline $2002 \mathrm{NY}_{31}$ & $11.50 \pm 0.02$ & & S \\
\hline (1917) Cuyo & $11.67 \pm 0.02$ & $\mathrm{Sl}^{(4)}$ & $S$ \\
\hline (25916) $2001 \mathrm{CP}_{44}$ & $13.94 \pm 0.04$ & & S \\
\hline $2002 \mathrm{QE}_{15}$ & $15.67 \pm 0.02$ & & $S$ \\
\hline $2002 \mathrm{DB}_{4}$ & $9.75 \pm 0.04$ & & $S$ \\
\hline $2002 \mathrm{~TB}_{58}$ & $18.29 \pm 0.11$ & & $\mathrm{D} ?$ \\
\hline (914) Palisana $^{\dagger}$ & $0.20 \pm 0.03$ & $\mathrm{CU}^{(1)}$ & $\mathrm{C} *$ \\
\hline \multirow[t]{2}{*}{ (393) Lampetia $^{\dagger}$} & $3.64 \pm 0.07$ & $\mathrm{C}^{(1)}$ & $\mathrm{C}^{*}$ \\
\hline & $0.30 \pm 0.04$ & & \\
\hline $1978 \mathrm{CA}$ & $12.22 \pm 0.03$ & $S^{(1)}$ & $S$ \\
\hline (4954) Eric & $15.04 \pm 0.06$ & $\mathbf{S}^{(1,2,3)}$ & $S$ \\
\hline
\end{tabular}

reddening". Spectral phase reddening was proven by observations of asteroids by Luu \& Jewitt (1990). They conclude that phase reddening corrections cannot be ignored for a proper comparison between NEAs and Main Belt asteroids.

A study of the dependency of the reflectance on the phase angle was made during the approach to (433) Eros by the NEAR spacecraft (Veverka et al. 2000; Bell et al. 2002; Clark et al. 2002b). These studies as well find a clear presence of phase reddening.

In addition, laboratory measurements by Gradie et al. (1980) and Gradie \& Veverka (1986) demonstrates further variations of spectral features with the phase angle. This is for instance the case for the shape and strength of the pyroxene absorption band centered at $\sim 0.9 \mu \mathrm{m}$.

The phase angle is considered as a correlating parameter for the wavelength-dependency in multiple light scattering theory(ies) (Lumme \& Bowell 1981, and private communication) ${ }^{1}$.

\footnotetext{
${ }^{1}$ In addition to the phase angle, the spectrum (encompassing both the slope and the spectral features) as well depends on further parameters (e.g. the incidence and emitting angle of the light with respect to the asteroid surface normal, the particle size, Gradie et al. 1980; Hapke 1981; Gradie \& Veverka 1986; and the surface roughness and porosity, Lumme \& Bowell 1981).
}

To date, no detailed studies are available on the influence of the phase reddening effect on ground-based asteroid reflectance spectra, including an examination of the dependency on taxonomic type. Thus, the implication of phase reddening on a thorough taxonomic classification scheme remains to be examined.

The taxonomic classification system proposed by Bus \& Binzel (2002a,b) suggests a division of the S class into 5 subgroups, and the $\mathrm{C}$ class into 4 subgroups, distinguished by variations in the spectral features. Recognizing the spectral slope as a dominating indicator of the spectral type, Bus \& Binzel decide to remove the spectral slope from the spectra, before the subsequent statistical analysis. This is accomplished by normalizing the spectra with the spectral slope measured over the entire asteroid spectra. We would expect this to work well for Main Belt asteroids, but for NEAs the phase reddening effect is more severe and needs to be taken into account. The phase reddening would according to the above cause different normalization levels for the spectra depending on the observational geometry, thus affecting the appearance of the spectral features. In addition, we believe that the variations in the spectral features among the subgroups of the Bus taxonomy are difficult to distinguish from the variations among the spectral features due to phase angle effects as described by Gradie \& Veverka (1986).

The Bus taxonomy was derived from studies of Main Belt asteroids, generally observed at relatively small phase angles. However, as NEAs are often studied at large phase angles, we find it necessary to consider phase angle effects in the Bus taxonomy.

As we in this work do not have data of the observed asteroids over more than one phase angle, and as we have found no comparable observations at other phase angles in the literature, we are not able to derive the individual phase variations. We hence find that the Tholen taxonomy, without the detailed characterization scheme, is the more suitable for our data.

In the following we will comment on our results for the observed asteroids:

(2329) Orthos. This Apollo asteroid was observed using a single $900 \mathrm{~s}$ exposure, and we find it to be an S-type.

$2002 A G_{29}$. For this Apollo asteroid we have both a singleexposure spectrum, as well as a two-part nodding spectrum. The nodding spectrum was originally suffering from an improper background removal as a result of varying sky background due to the appearance of cirrus clouds. Hence, instead of the direct subtraction of the $A B$ spectra, the background is removed by the standard method applied on each of the spectra, followed by an averaging of the spectra before division with the Solar Analog. A comparison between the single-exposure and the nodded spectrum shows similar spectral shape, and we have classified the asteroid as an S-type.

(4957) Brucemurray. This S-type Amor spectrum is extracted from only the A-part of the AB-pair. The classification is in agreement with the one of Angeli \& Lazarro (2002). 
(35107) $1991 \mathrm{VH}$. The spectrum of this S-type Apollo asteroid was extracted from a single pair of exposures. The asteroid has been classified as an Sk by Binzel et al. (2004).

$2002 N Y_{31}$. From the obtained data (ABBA nodding pairs) we conclude that this Amor asteroid is an S-type.

(1917) Cuyo. We found this Amor asteroid to be an S-type, the spectrum was extracted from one pair of nodded spectra. The asteroids has been classified as an Sl type by Binzel et al. (2004).

(25916) $2001 \mathrm{CP}_{44}$. An S-type Amor asteroid, however, rather flat beyond $8000 \AA$. The shown spectrum is an average of a pair of spectra.

$2002 Q E_{15}$. We notice the presence of a broad absorption band starting at $7500 \AA$. We have classified this Amor asteroid as an S-type, based on a pair of spectra.

$2002 \mathrm{DB}_{4}$. This Aten asteroid was observed at a phase angle of $62.3^{\circ}$. The spectrum was extracted from a single pair of nodded spectra, and we find the asteroid to be an S-type (see also the discussion in Sect. 4).

$2002 T B_{58}$. The Amor asteroid $2002 T B_{58}$ has a slope of $S^{\prime}=18.3 \% / 1000 \AA$, which is quite extreme. The spectrum shows a discontinuity at $6800 \AA$. We further discuss the classification of this asteroid in Sect. 4.

1978 CA. The shown spectrum is the average of six individual exposures of one hour total exposure time. We find this Apollo asteroid to be an S-type, in disagreement with the tabulation found in Binzel et al. (2002) who lists this asteroid to be an M-type. However, our result is in agreement with Tholen (1989).

(4954) Eric. The shown spectrum was computed by using four exposures, and we find it to be an S-type. The spectrum is in good qualitative agreement with the spectrum presented by Lazzarin et al. (1997), also arriving at an S-type classification. This Amor asteroid was also classified as an S-type by Angeli \& Lazzaro (2002).

The Main Belt asteroids. The two C-type asteroids, (393) Lampetia and (914) Palisana, do not belong to the NEA population. They were observed as part of the experiments with the differential guiding at the Danish telescope. They are located at the innermost part of the asteroid Main Belt, with their perihelion distance $q$ is stated in the second column of Table 1 . In both cases, the spectra were extracted from single exposures. The best guess classifications, based on the truncated spectra, are in agreement with the literature (Table 2).

One of the asteroids, (914) Palisana, was previously suspected to have indications of phyllosilicates, due to the presence of a broad but weak absorption feature centered around $7000 \AA$ (Fitzsimmons et al. 1994). Dotto et al. (2002) performed

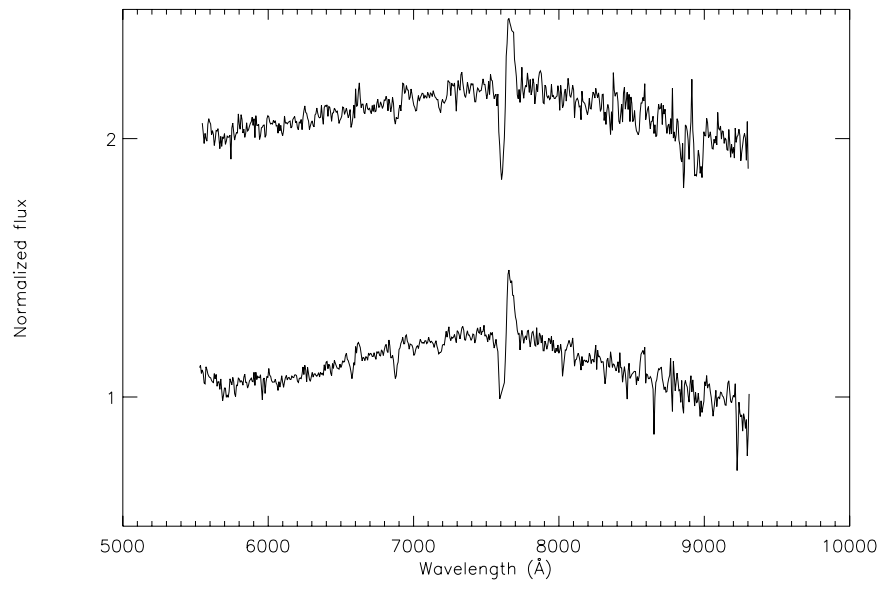

Fig. 4. Normalized spectra of (1917) Cuyo, obtained from the same set of $A B$ spectra, but reduced with two different methods. For the lower spectrum, the background removal was obtained by applying the nodding method, using individual sky sub-frames. The upper spectrum is an average of two spectra for which the sky background correction has been achieved using the standard method.

observations of the asteroid with the ISO satellite in the infrared region, but were not able to identify any features indicative of phyllosilicates. Unfortunately the spectrum presented here is distorted beyond $7000 \AA$, preventing a detailed analysis of the spectrum. From the immediate properties of the spectrum, we do not see any indication of an absorption band.

\section{Discussion and conclusions}

With the use of the nodding technique we were able to reduce the requirements on the target tracking capabilities of the telescopes. In addition, we find that the method improves the data reduction, in terms of an in general improved sky background removal. An example is shown in Fig. 4, where we have reduced the two AB spectra of (1917) Cuyo using the nodding method of individual sky sub-frames (lower spectrum) and the standard sky removal using a polynomial fit (upper spectrum). The latter was obtained by reducing each spectrum individually, removing the background from each spectrum, and by averaging the two final spectra. The lower spectrum is the same as in Fig. 2, reduced by using the nodding method on a pair of spectra, leading typically to less spectral noise, especially in the red part of the spectrum, when compared to the outcome of the standard method.

With the exception of $2002 \mathrm{~TB}_{58}$, all of the NEAs observed within this study can be classified as S-type asteroids. In contrast, the C-type asteroids are the most abundant in the Main Belt, only surpassed by the S-types in the innermost region (Mothé-Diniz et al. 2003). While our observations do not represent a systematic spectroscopic survey, that can be analyzed in terms of observational biases, we however do find a correspondence to the conclusion by Luu \& Jewitt (1989) that S-type asteroids are overabundant among the NEAs compared to the Main Belt. Various explanations have been suggested (cf. Binzel et al. 2002, for an overview): selection effects due to the low albedo of C-type asteroids, and faster 
decreasing brightness with increasing phase angle, compared to S-types. However, these effects probably do not stand alone. The presence of the 3:1 resonance in the inner Main Belt, suspected to be a major source for the replenishment of the NEAs (Morbidelli \& Nesvorný 1999), consistent with the relatively low density of asteroids in this region (Lagerkvist \& Lagerros 1997). As the inner Main Belt is mostly dominated by S-type asteroids, it thus seems likely that these asteroids do tend to be more abundant among the NEAs, which is also indicated from our observations.

The asteroid $2002 \mathrm{~TB}_{58}$ presents a particular case, with a slope of $S^{\prime}=18.3 \% / 1000 \AA$, observed at $\alpha=60^{\circ}$. A slope of the same size was found by Luu \& Jewitt (1990) for the asteroid (2927) Alamosa at a phase angle of $25.1^{\circ}$. They suggested a general phase reddening correction of $\gamma=0.2 \% / 1000 \AA / \mathrm{deg}$ for phase angles $0^{\circ}<\alpha \leq 40^{\circ}$, with $\gamma=0.0 \% / 1000 \AA / \mathrm{deg}$ for $\alpha>40^{\circ}$.

If we apply the estimate for the phase correction suggested by Luu \& Jewitt (1990), we find a phase-corrected slope of $S_{\text {cor }}^{\prime}=S^{\prime}-\gamma \alpha=10.3$. In this case the asteroid would then be classified as a typical D-type asteroid, however, as the phase correction is uncertain the asteroid could as well be of type $\mathrm{C}$ or X (E, M or P, depending on the albedo). We therefore conclude that the classification should be confirmed by a further spectrum to be obtained at a smaller phase angle, possibly in combination with an explicit determination of the phase reddening for this asteroid.

The spectrum of $2002 \mathrm{DB}_{4}$ was obtained at nearly the same phase angle as $2002 \mathrm{~TB}_{58}$. Applying the same Luu \& Jewitt phase correction for $2002 \mathrm{DB}_{4}$ as for $2002 \mathrm{~TB}_{58}$ results in a slope of $S_{\text {cor }}^{\prime}=1.8$, i.e. the slope of a $\mathrm{C}$ or X type asteroid. As the spectra of these asteroids are in general almost featureless in the considered wavelength region, this implies a contradiction as the overall shape of the spectrum resembles an $\mathrm{S}$ type. If we instead apply a phase-correction of $\gamma=0.067 \% / 1000 \AA / \mathrm{deg}$ measured by Nathues (2000), valid for $S$-type asteroids in the range 0.4 to $0.7 \mu \mathrm{m}$ and a phase angle range of $0^{\circ}<\alpha \leq 27^{\circ}$, we arrive at a slope of $S_{\text {cor }}^{\prime}=5.6$, which more readily conform with the S-type nature of the spectrum.

From these considerations we thus arrive at a situation where we cannot argue for the application of one or the other phase reddening correction, as in either case one method is preferred to the other, according to a prejudice on the most realistic outcome of the phase correction. We agree with Luu \& Jewitt (1990) that phase reddening has an important influence on the spectral slope, but it is not clear how this correction can be generalized to a wide number of asteroids. Hence this issue warrants further examinations. For the outermost consequences of the taxonomic classification of NEAs, we in addition find it necessary to investigate the strength of the phase reddening effect on the absorption bands of the spectra.

Acknowledgements. R.M. is obliged to Ola Karlsson for many discussions. We have made use of the SIMBAD database, operated at CDS, Strasbourg, France and of the European Asteroid Research Node (EARN), maintained by G. Hahn, DLR, Berlin.

This project was supported by the Danish Natural Science Research Council through its Centre for Ground-Based Observational Astronomy (IJAF).

\section{References}

Angeli, C. A., \& Lazzaro, D. 2002, A\&A, 391, 757

Bell III, J. F., Izenberg, N. I., Lucey, P. G., et al. 2002, Icarus, 155, 119

Binzel, R. P., \& Xu, S. 1993, Science, 260, 186

Binzel, R. P., Bus, S. J., Burbine, T. H., et al. 1996, Science, 273, 946

Binzel, R. P., Harris, A. W., Bus, S. J., et al. 2001, Icarus, 151, 139

Binzel, R. P., Lupishko, D. F., Di Martino, M., et al. 2002, in Asteroids III, ed. W. F. Bottke Jr., A. Cellino, P. Paolicchi, \& R. P. Binzel (Univ. Ariz. Press)

Binzel, R. P., Rivkin, A. S., Stuart, J. S., et al. 2004, Icarus, 170, 259

Bottke Jr., W. F., Vokrouhlický, D., Rubincam, D. P., et al. 2002, in Asteroids III, ed. W. F. Bottke Jr., A. Cellino, P. Paolicchi, \& R. P. Binzel (Univ. Ariz. Press)

Bus, S. J., \& Binzel, R. P. 2002a, Icarus, 158, 106

Bus, S. J., \& Binzel, R. P. 2002b, Icarus, 158, 146

Clark, B. E., Hapke, B., Pieters, C., et al. 2002a, in Asteroids III, ed. W. F. Bottke Jr., A. Cellino, P. Paolicchi, \& R. P. Binzel (Univ. Ariz. Press)

Clark, B. E., Helfenstein, P., Bell III, J. F., et al. 2002b, Icarus, 155, 189

Davis, D. R., Durda, D. D., Marzari, F., et al. 2002, in Asteroids III, ed. W. F. Bottke Jr., A. Cellino, P. Paolicchi, \& R. P. Binzel (The Univ. Ariz. Press)

Dotto, E., Barucci, M. A., Müller, T. G., et al. 2002, A\&A, 393, 1065

Fitzsimmons, A., Dahlgren, M., Lagerkvist, C.-I., et al. 1994, A\&A, 282,634

Gradie, J., \& Veverka, J. 1986, Icarus, 66, 455

Gradie, V., Veverka, J., \& Buratti, B. 1980, Proc. Lunar Planet. Sci. Conf. 11th, 799

Haack, H., Michelsen, R., Andersen, A. C., et al. 2002, in Proc. of ACM 2002, ESA Special Publications SP-500

Hapke, B. 1981, J. Geophys. Res., 86, 3039

Hardorp, J. 1978, A\&A, 63, 383

Lagerkvist, C.-I., \& Lagerros, J. S. V. 1997, Astron. Nachr., 318, 391

Landolt, A. U. 1973, AJ, 78, 959

Lazzarin, M., Di Martino, M., Barucci, M. A., et al. 1997, A\&A, 327, 388

Lumme, K., \& Bowell, E. 1981, AJ, 86, 1694

Lupishko, D. F., \& Di Martino, M. 1998, Planet. Space Sci., 46, 47

Luu, J., \& Jewitt, D. 1989, AJ, 98, 1905

Luu, J. X., \& Jewitt, D. C. 1990, AJ, 99, 1985

Meibom, A., \& Clark, B. E. 1999, Meteorit. Planet. Sci., 34, 7

Morbidelli, A., \& Nesvorný, D. 1999, Icarus, 139, 295

Morrison, D., Harris, A. W., Sommer, G., et al. 2002, in Asteroids III, ed. W. F. Bottke Jr., A. Cellino, P. Paolicchi, \& R. P. Binzel (Univ. Ariz. Press)

Mothé-Diniz, T., Carvano, J. M., \& Lazzaro, D. 2003, Icarus, 162, 10

Nathues, A. 2000, Studie der Eunomia Asteroidenfamilie mittels Spektroskopie, Ph.D. Thesis, DLR, Berlin

Nesvorný, D., Ferraz-Mello, S., Holman, M., et al. 2002, in Asteroids III, ed. W. F. Bottke, A. Cellino, P. Paolicchi, \& R. P. Binzel (Univ. Ariz. Press)

Storm, J. 1996, Differential guiding at the Danish 1.54m, memorandum, ESO

Tholen, D. J. 1984, Asteroid taxonomy from cluster analysis of photometry, Ph.D. Thesis (University of Arizona)

Tholen, D. J. 1989, in Asteroids II, ed. R. P. Binzel et al. (Univ. Ariz. Press)

Tholen, D. J., \& Barucci, M. A. 1989, in Asteroids II, ed. R. P. Binzel et al. (Univ. Ariz. Press)

Tüg, H. 1977, Messenger, 11, 7

Veverka, J., Robinson, M., Thomas, P., et al. 2000, Science, 289, 2088 